Supporting Information

\title{
Extending Stannyl Anion Chemistry to the Actinides: Synthesis and Characterization of a Uranium-Tin Bond
}

\author{
Matthew S. Winston ${ }^{1}$, Enrique R. Batista ${ }^{2}$, Ping Yang ${ }^{2}$, Aaron M. Tondreau ${ }^{1}$, and James M. \\ Boncella $^{1 *}$ \\ ${ }^{1}$ Los Alamos National Laboratory, Chemistry Division, Los Alamos, New Mexico 87545, United States \\ ${ }^{2}$ Los Alamos National Laboratory, Theoretical Division, Los Alamos, New Mexico 87545, United States
}

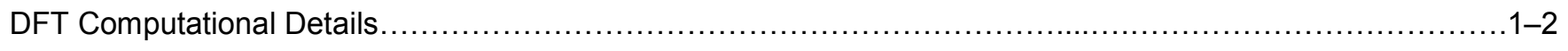

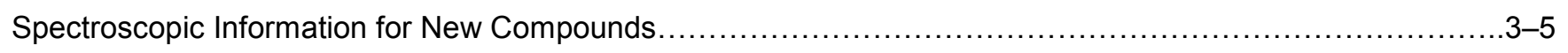

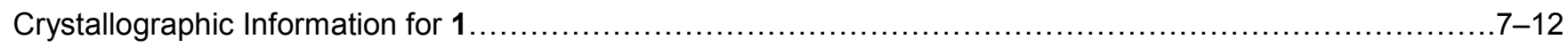

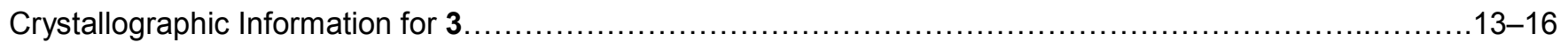

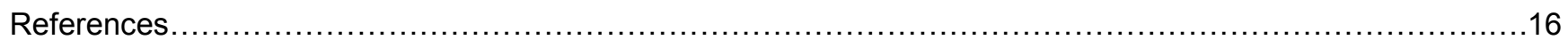


Simulation details: Hybrid DFT using Truhlar's functional M06. ${ }^{1}$ Optimized molecular structure from crystal provided. SDD relativistic pseudopotentials were used to represent the $U$ and $\mathrm{Sn} .6-31+\mathrm{g}^{*}$ basis set was used for all other ligand atoms.

Structural parameters of optimized structure differ by up to $2 \%$ from experimental measurement.

$\begin{array}{lrrr} & \text { Crystal } & \text { DFT-M06 } & \text { Error } \\ \text { U-Sn } & 3.31303 & 3.38347 & 2.13 \% \\ \text { U-N(1) } & 2.2266 & 2.22304 & -0.16 \% \\ \text { U-N(2) } & 2.23671 & 2.24232 & 0.25 \% \\ \text { U-N(4) } & 2.24646 & 2.24734 & 0.04 \% \\ \text { U-N(3) } & 2.63539 & 2.69144 & 2.13 \%\end{array}$

Electronic state: $U(I V) f^{2}$

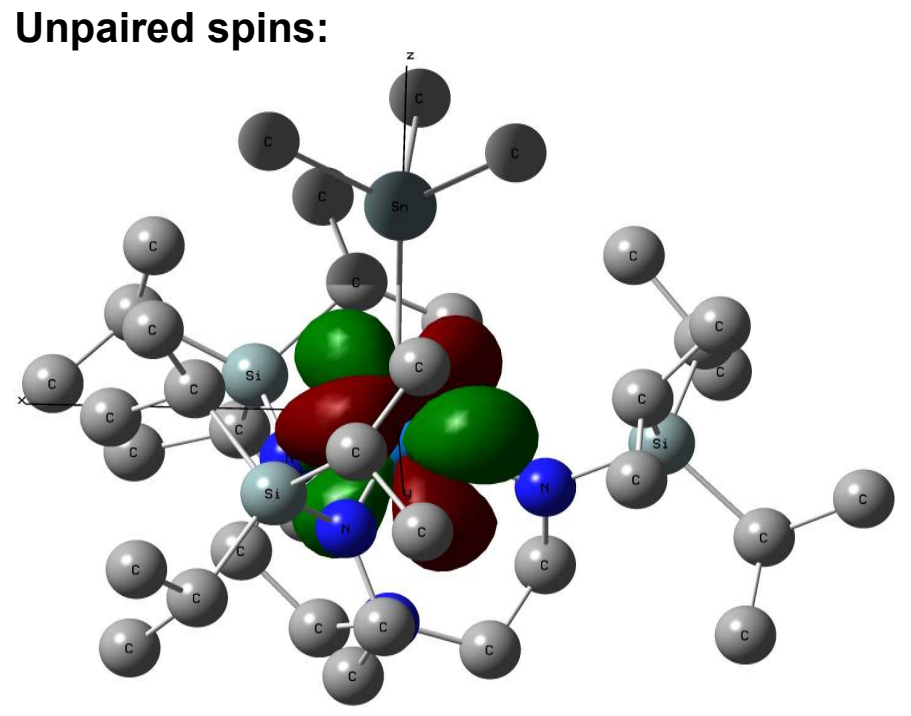

Non-bonding 1: $96 \% 5 f, 3 \% d$

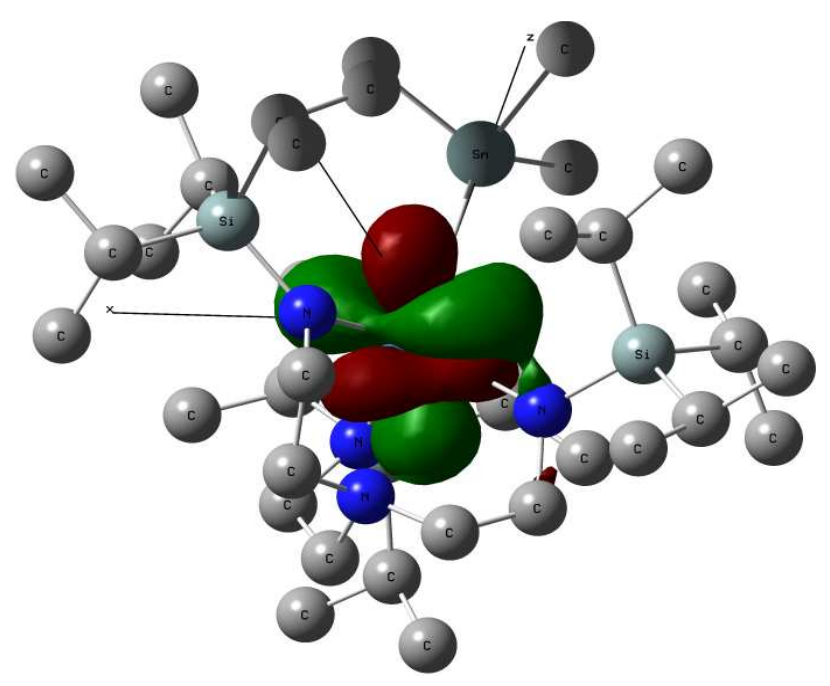

Non-bonding 2: $96 \% 5 f, 3 \% d$ 


\section{Natural Bond Orbital analysis:}

The bonding orbital wave function is composed of a $25 \%$ and $75 \%$ contributions from uranium and tin, respectively.

$$
\Psi_{\mathrm{NBO}}=0.5007 \varphi_{\mathrm{U}}+0.8656 \varphi_{\mathrm{Sn}}
$$

The bond is polarized with significant charge transfer from $U$ to $S n$. NBO analysis identifies a single bond between $U$ and $S n$ of sigma character.

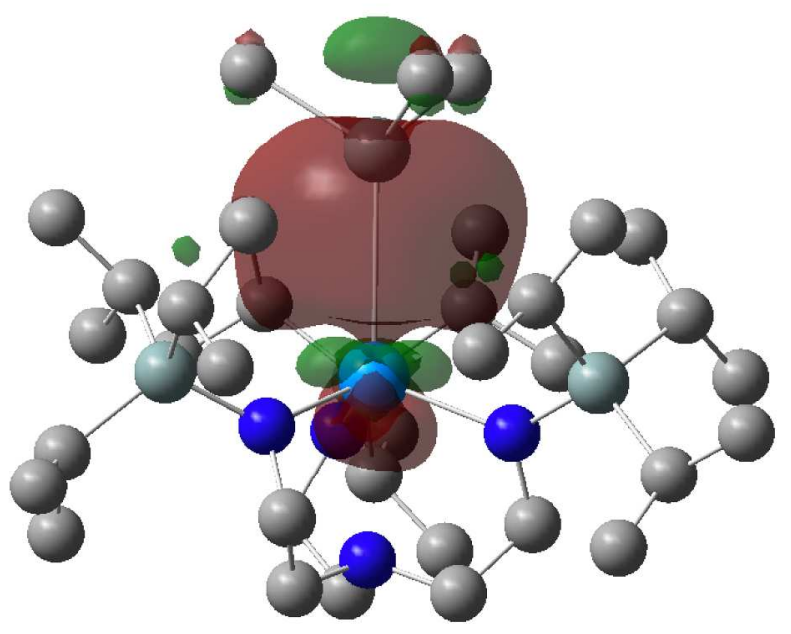

Uranium component: $26.4 \% s, 58.2 \% d$, and $14.2 \% f$

Sn component: $41.5 \% s, 58.5 \% p$ 


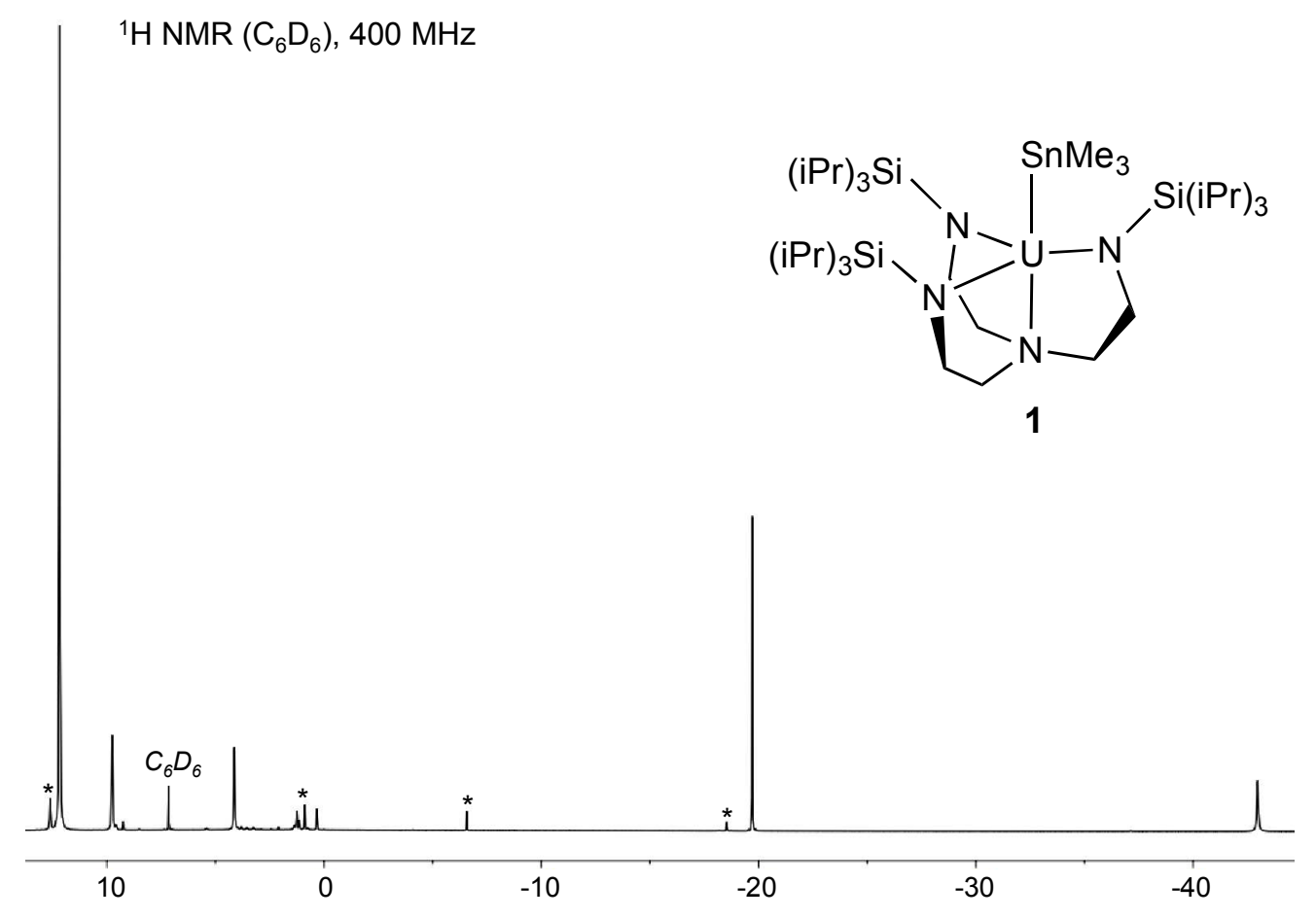

Figure $\mathrm{S} 1 .{ }^{1} \mathrm{H}(400 \mathrm{MHz}) \mathrm{NMR}$ spectrum of 1 in $\mathrm{C}_{6} \mathrm{D}_{6}$. ( ${ }^{*}$ impurities or hexane)

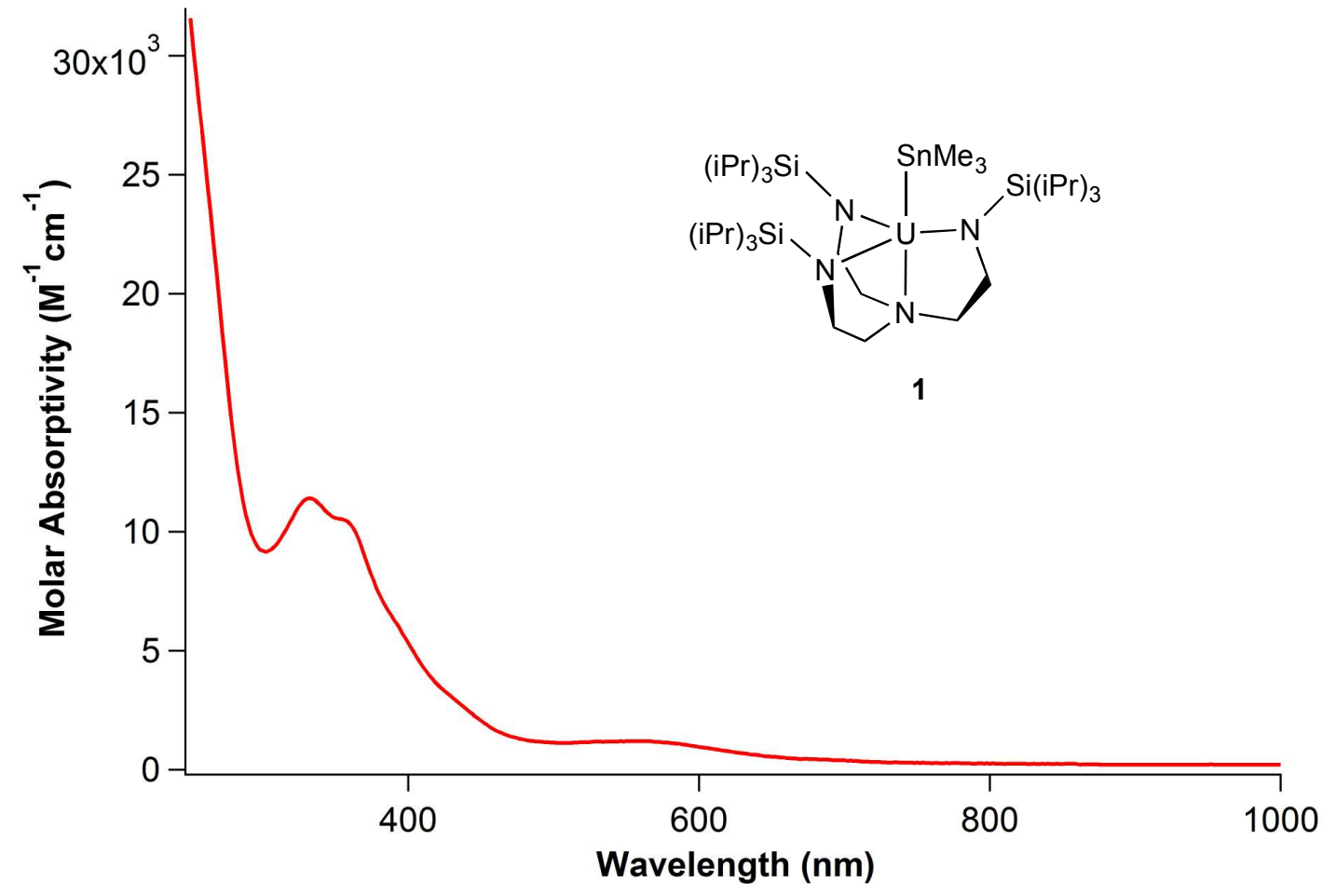

Figure S2. UV/vis spectrum of 1 in hexane $(0.34 \mathrm{mM})$ 


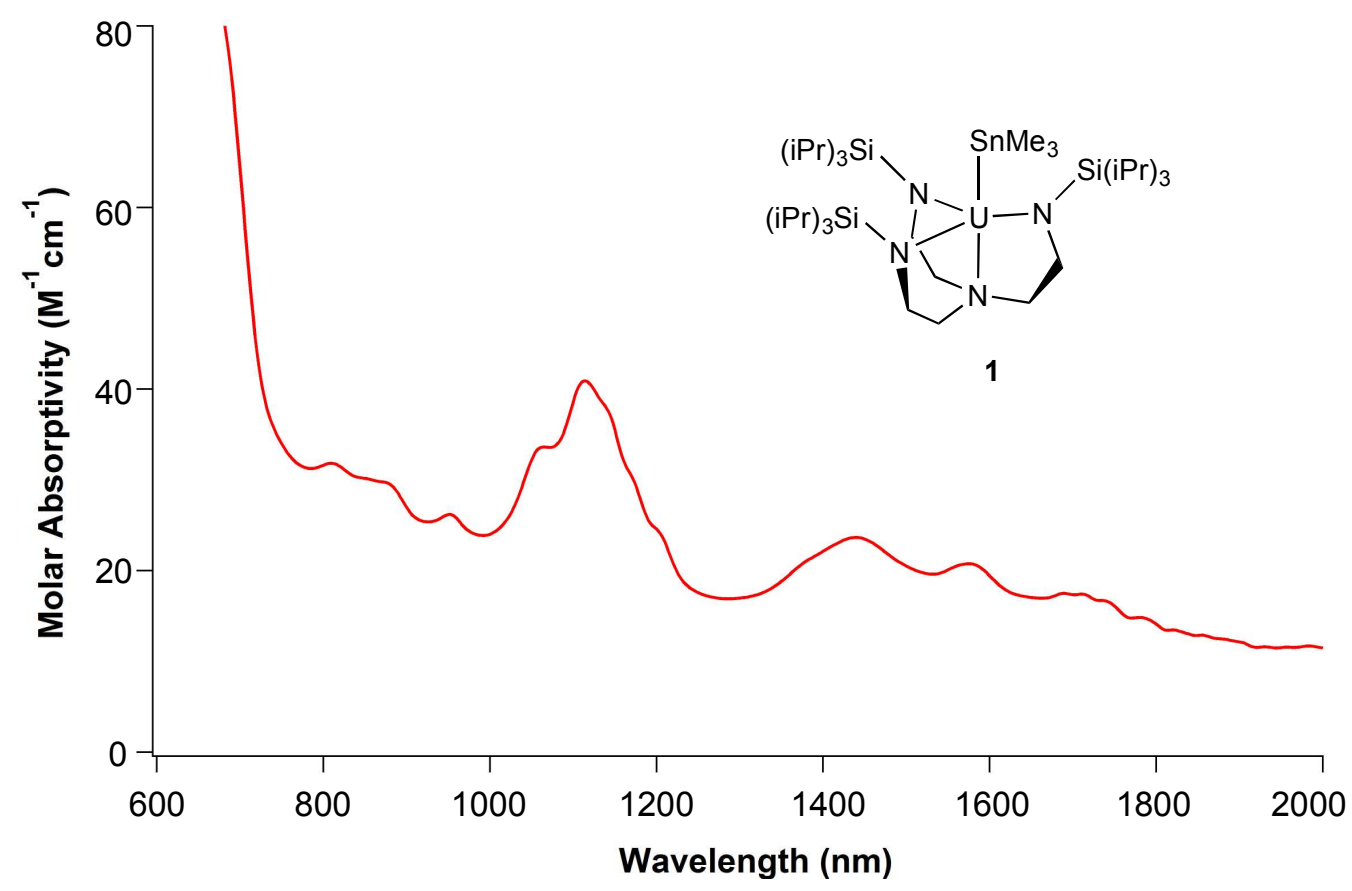

Figure S3. NIR spectrum of 1 in hexane (17 mM)

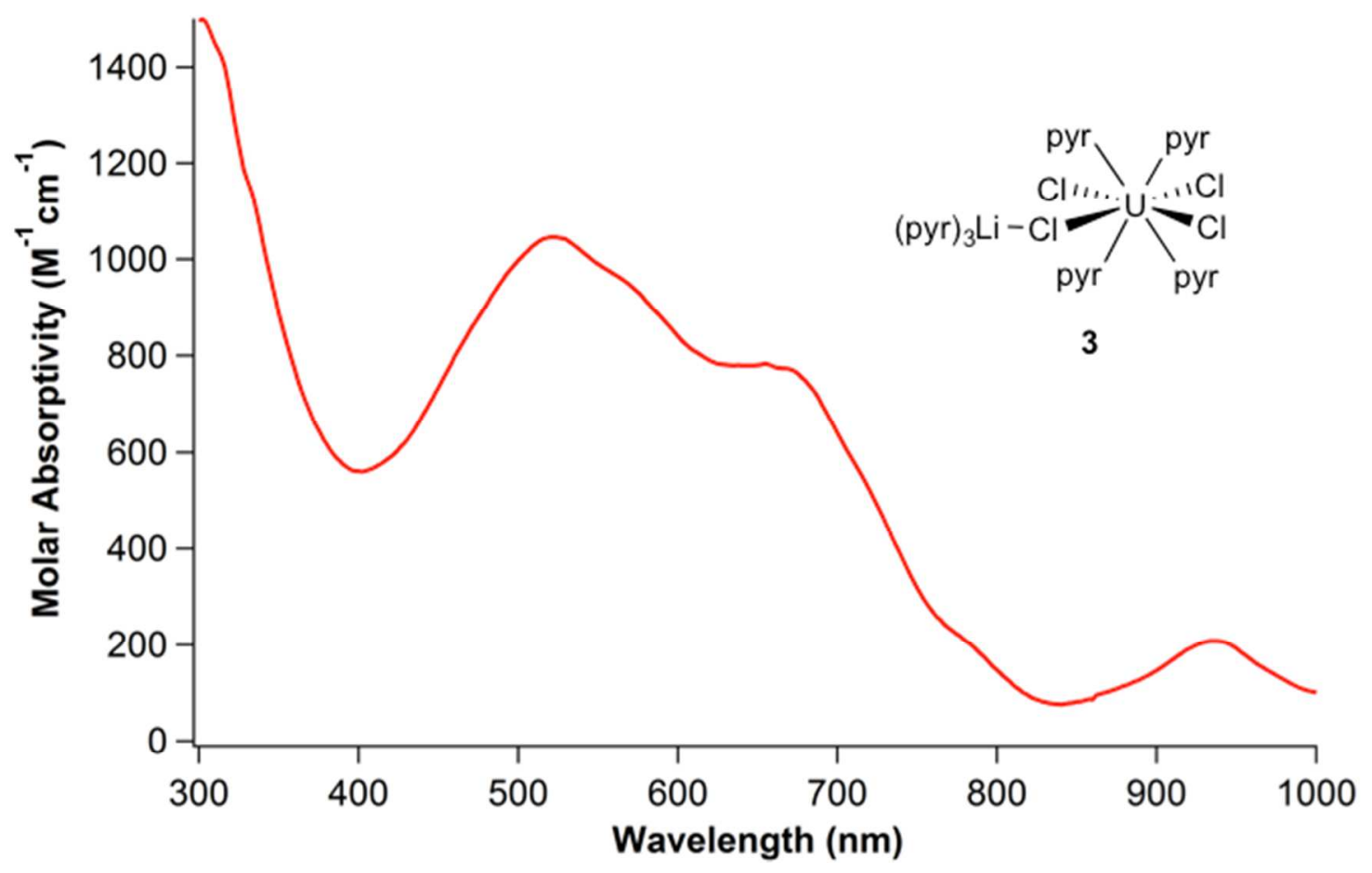

Figure S4. UV/vis spectrum of $\mathbf{3}$ in pyridine $(2.7 \mathrm{mM})$ 


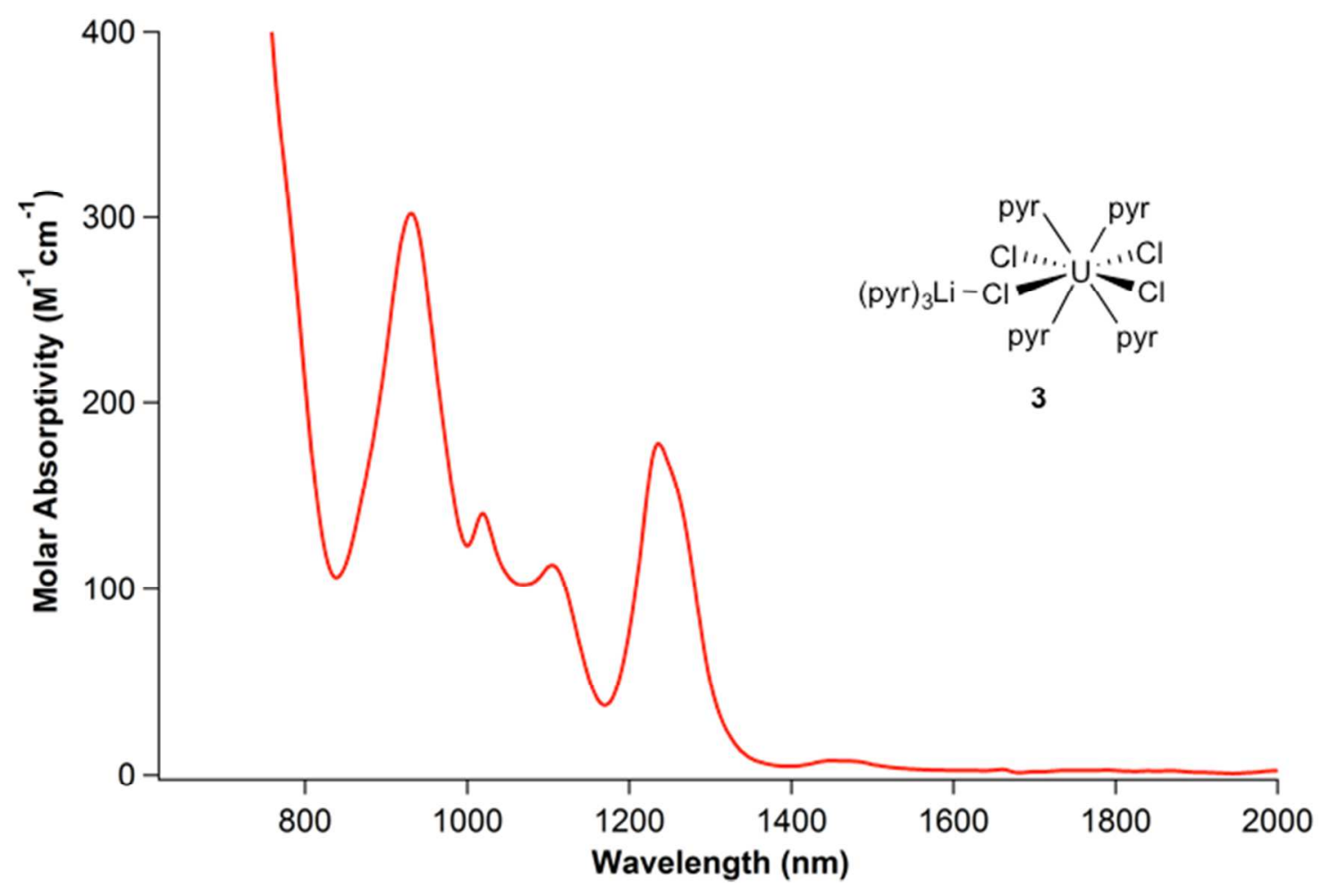

Figure S5. NIR spectrum of 3 in pyridine $(27 \mathrm{mM})$ 
Table S1. Crystal and refinement data for 1 and 3.

\begin{tabular}{|c|c|c|}
\hline & 1 & 3 \\
\hline empirical formula & $\mathrm{C}_{36} \mathrm{H}_{84} \mathrm{~N}_{4} \mathrm{Si}_{3} \mathrm{SnU}$ & $\mathrm{C}_{35} \mathrm{H}_{35} \mathrm{Cl}_{4} \mathrm{LiN}_{7} \mathrm{U}$ \\
\hline formula wt & 1014.09 & 940.48 \\
\hline $\mathrm{T}(\mathrm{K})$ & 100 & 100 \\
\hline$a(\AA)$ & $10.6634(7)$ & $17.762(4)$ \\
\hline$b(\AA)$ & $25.0575(16)$ & $11.798(3)$ \\
\hline$c(\AA)$ & $18.0413(12)$ & $19.524(4)$ \\
\hline$\alpha(\mathrm{deg})$ & 90 & 90 \\
\hline$\beta$ (deg) & $100.015(1)$ & $113.735(2)$ \\
\hline$\gamma(\mathrm{deg})$ & 90 & 90 \\
\hline $\mathrm{V}\left(\AA^{3}\right)$ & $4557.7(5)$ & $3745.3(14)$ \\
\hline $\mathrm{Z}$ & 4 & 4 \\
\hline crystal system & monoclinic & monoclinic \\
\hline space group & $\mathrm{P} 2_{1} / n$ & $\mathrm{P} 2_{1} / n$ \\
\hline density $\left(\mathrm{g} \mathrm{cm}^{-3}\right)$ & 1.472 & 1.668 \\
\hline$\theta_{\max }(\mathrm{deg})$ & 28.280 & 28.280 \\
\hline$\mu\left(\mathrm{mm}^{-1}\right)$ & 4.202 & 4.654 \\
\hline GOF & 1.032 & 1.278 \\
\hline $\mathrm{R} 1^{\mathrm{a}}$ & 0.0265 & 0.0645 \\
\hline wR2 $\left(I>2 \sigma(I)^{\mathrm{b}}\right.$ & 0.0663 & 0.1629 \\
\hline $\mathrm{a} \mathrm{R} 1=\boldsymbol{S}\|F|-| F\| /$ & & \\
\hline
\end{tabular}


Crystallographic Information for ( $\left.{ }^{\mathrm{TIPS}} \mathrm{TREN}\right) \mathrm{U}\left(\mathrm{SnMe}_{3}\right)$ (1).

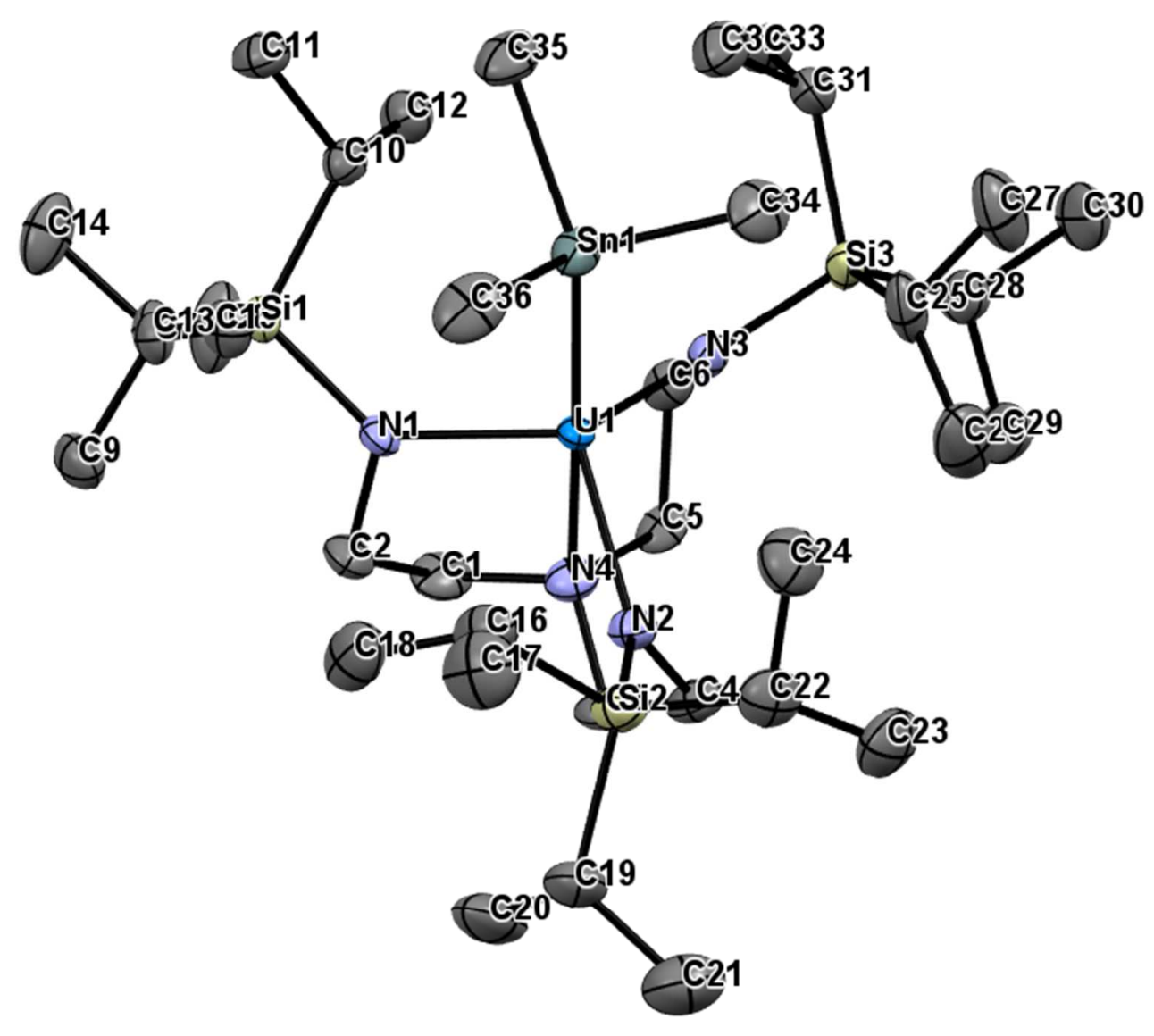

Figure S6. Thermal ellipsoid representations of 1 at the $50 \%$ probability level, from two perspectives. 
Table S2. Crystal parameters for 1.

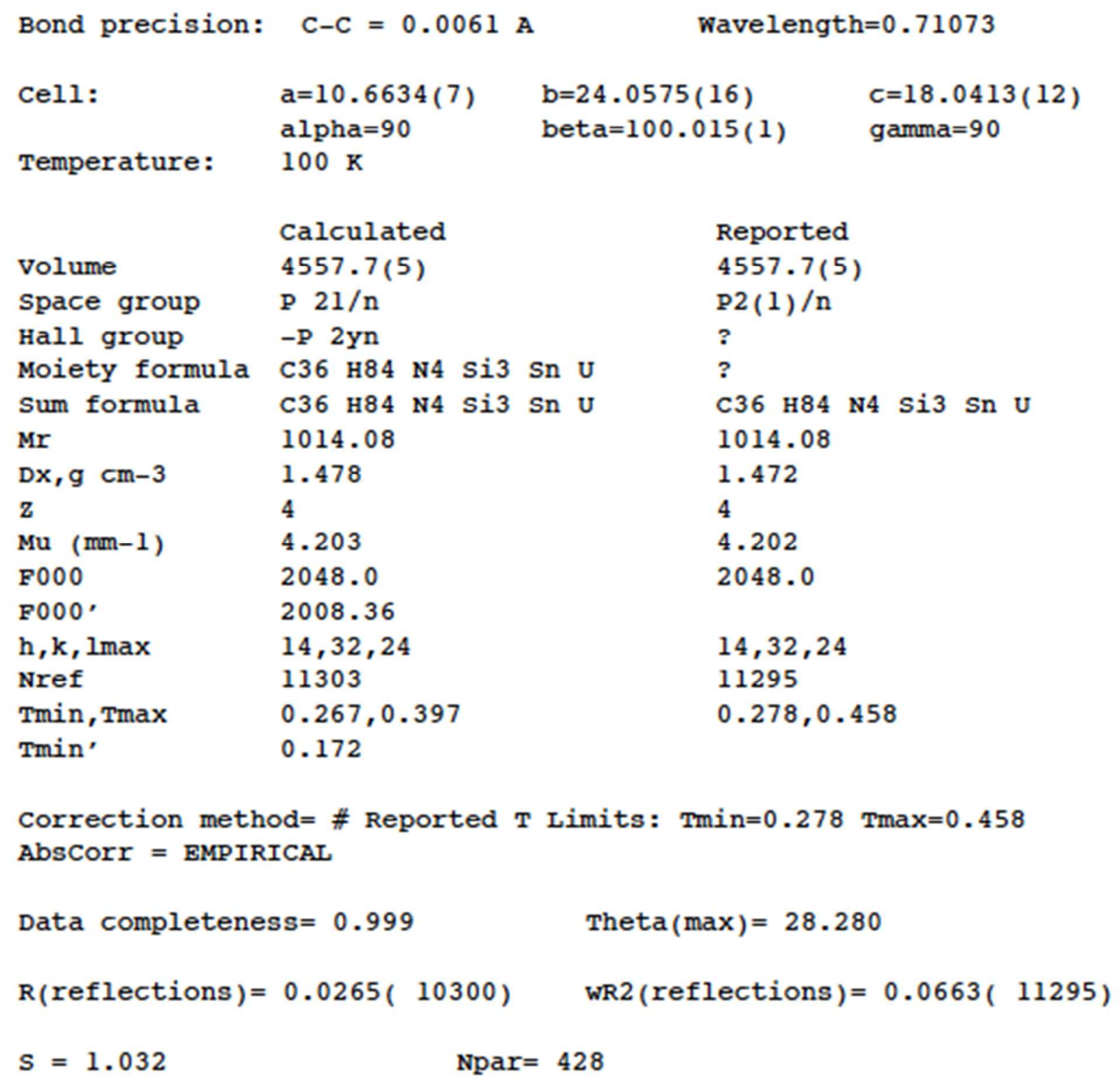

Table S3. Bond lengths for 1 . 
C1 N4 1.482(5)

C1 C2 1.508(5)

C1 H1A 0.9900

C1 H1B 0.9900

C2 N1 1.484(4)

C2 H2A 0.9900

C2 H2B 0.9900

C3 N4 1.479(4)

C3 C4 1.509(5)

C3 H3A 0.9900

C3 H3B 0.9900

C4 N2 1.487(4)

C4 H4A 0.9900

C4 H4B 0.9900

C5 N4 1.469(5)

C5 C6 1.530(5)

C5 H5A 0.9900

C5 H5B 0.9900

C6 N3 1.485(4)

C6 H6A 0.9900

C6 H6B 0.9900

C7 C9 1.525(5)

C7 C8 1.530(5)

C7 Si1 1.899(3)

C7 H7 1.0000

C8 H8A 0.9800

C8 H8B 0.9800

C8 H8C 0.9800

C9 H9A 0.9800

C9 H9B 0.9800

C9 H9C 0.9800

C10 C11 1.539(5)

C10 C12 1.544(5)

C10 Si1 1.891(3)

C10 H10 1.0000

C11 H11A 0.9800

C11 H11B 0.9800

C11 H11C 0.9800

C12 H12A 0.9800

C12 H12B 0.9800

C12 H12C 0.9800

C13 C14 1.528(5)

C13 C15 1.534(5)

C13 Si1 1.904(3)

C13 H13 1.0000

C14 H14A 0.9800

C14 H14B 0.9800

C14 H14C 0.9800

C15 H15A 0.9800
C15 H15B 0.9800

C15 H15C 0.9800

C16 C17 1.545(6)

C16 C18 1.556(7)

C16 Si2 1.884(4)

C16 H16 1.0000

C17 H17A 0.9800

C17 H17B 0.9800

C17 H17C 0.9800

C18 H18A 0.9800

C18 H18B 0.9800

C18 H18C 0.9800

C19 C20 1.516(6)

C19 C21 1.563(7)

C19 Si2 1.908(4)

C19 H19 1.0000

C20 H20A 0.9800

C20 H20B 0.9800

$\mathrm{C} 20 \mathrm{H} 20 \mathrm{C} 0.9800$

C21 H21A 0.9800

C21 H21B 0.9800

$\mathrm{C} 21 \mathrm{H} 21 \mathrm{C} 0.9800$

C22 C23 1.523(6)

C22 C24 1.529(6)

C22 Si2 1.895(4)

$\mathrm{C} 22 \mathrm{H} 221.0000$

C23 H23A 0.9800

C23 H23B 0.9800

C23 H23C 0.9800

C24 H24A 0.9800

C24 H24B 0.9800

C24 H24C 0.9800

C25 C26 1.545(7)

C25 C27 1.544(6)

C25 Si3 1.879(4)

$\mathrm{C} 25 \mathrm{H} 251.0000$

C26 H26A 0.9800

C26 H26B 0.9800

C26 H26C 0.9800

C27 H27A 0.9800

C27 H27B 0.9800

C27 H27C 0.9800

C28 C29 1.527(6)

C28 C30 1.527(6)

C28 Si3 1.907(4)

$\mathrm{C} 28 \mathrm{H} 281.0000$

C29 H29A 0.9800

C29 H29B 0.9800

C29 H29C 0.9800 
C30 H30A 0.9800

C30 H30B 0.9800

C30 H30C 0.9800

C31 C32 1.532(6)

C31 C33 1.533(5)

C31 Si3 1.892(4)

C31 H31 1.0000

C32 H32A 0.9800

C32 H32B 0.9800

C32 H32C 0.9800

C33 H33A 0.9800

C33 H33B 0.9800

C33 H33C 0.9800

C34 Sn1 2.186(4)

C34 H34A 0.9800

C34 H34B 0.9800

C34 H34C 0.9800

Table S4. Bond angles for 1 .

N4 C1 C2 109.4(3)

N4 C1 H1A 109.8

C2 C1 H1A 109.8

N4 C1 H1B 109.8

C2 C1 H1B 109.8

H1A C1 H1B 108.2

N1 C2 C1 108.8(3)

N1 C2 H2A 109.9

C1 C2 H2A 109.9

N1 C2 H2B 109.9

C1 C2 H2B 109.9

H2A C2 H2B 108.3

N4 C3 C4 109.0(3)

N4 C3 H3A 109.9

C4 C3 H3A 109.9

N4 C3 H3B 109.9

C4 C3 H3B 109.9

H3A C3 H3B 108.3

N2 C4 C3 108.8(3)

N2 C4 H4A 109.9

C3 C4 H4A 109.9

N2 C4 H4B 109.9

C3 C4 H4B 109.9

H4A C4 H4B 108.3

N4 C5 C6 109.0(3)

N4 C5 H5A 109.9

C6 C5 H5A 109.9

N4 C5 H5B 109.9

C6 C5 H5B 109.9
C35 Sn1 2.184(4)

C35 H35A 0.9800

C35 H35B 0.9800

C35 H35C 0.9800

C36 Sn1 2.219(5)

C36 H36A 0.9800

C36 H36B 0.9800

C36 H36C 0.9800

N1 Si1 1.754(3)

N1 U1 2.247(3)

N2 Si2 1.755(3)

N2 U1 2.234(3)

N3 Si3 1.752(3)

N3 U1 2.225(3)

N4 U1 2.636(3)

Sn1 U1 3.3129(3)

H5A C5 H5B 108.3

N3 C6 C5 108.8(3)

N3 C6 H6A 109.9

C5 C6 H6A 109.9

N3 C6 H6B 109.9

C5 C6 H6B 109.9

H6A C6 H6B 108.3

C9 C7 C8 110.3(3)

C9 C7 Si1 112.1(2)

C8 C7 Si1 111.9(2)

C9 C7 H7 107.4

C8 C7 H7 107.4

Si1 C7 H7 107.4

C7 C8 H8A 109.5

C7 C8 H8B 109.5

H8A C8 H8B 109.5

C7 C8 H8C 109.5

H8A C8 H8C 109.5

H8B C8 H8C 109.5

C7 C9 H9A 109.5

C7 C9 H9B 109.5

H9A C9 H9B 109.5

C7 C9 H9C 109.5

H9A C9 H9C 109.5

H9B C9 H9C 109.5

C11 C10 C12 108.6(3)

C11 C10 Si1 114.3(2)

C12 C10 Si1 113.5(2)

C11 C10 H10 106.6 
C12 C10 H10 106.6

Si1 C10 H10 106.6

C10 C11 H11A 109.5

C10 C11 H11B 109.5

H11A C11 H11B 109.5

C10 C11 H11C 109.5

H11A C11 H11C 109.5

H11B C11 H11C 109.5

C10 C12 H12A 109.5

C10 C12 H12B 109.5

H12A C12 H12B 109.5

C10 C12 H12C 109.5

H12A C12 H12C 109.5

H12B C12 H12C 109.5

C14 C13 C15 109.1(3)

C14 C13 Si1 111.8(3)

C15 C13 Si1 117.2(3)

C14 C13 H13 106.0

C15 C13 H13 106.0

Si1 C13 H13 106.0

C13 C14 H14A 109.5

C13 C14 H14B 109.5

H14A C14 H14B 109.5

C13 C14 H14C 109.5

H14A C14 H14C 109.5

H14B C14 H14C 109.5

C13 C15 H15A 109.5

C13 C15 H15B 109.5

H15A C15 H15B 109.5

C13 C15 H15C 109.5

H15A C15 H15C 109.5

H15B C15 H15C 109.5

C17 C16 C18 109.2(4)

C17 C16 Si2 113.2(3)

C18 C16 Si2 112.5(3)

C17 C16 H16 107.2

C18 C16 H16 107.2

Si2 C16 H16 107.2

C16 C17 H17A 109.5

C16 C17 H17B 109.5

H17A C17 H17B 109.5

C16 C17 H17C 109.5

H17A C17 H17C 109.5

H17B C17 H17C 109.5

C16 C18 H18A 109.5

C16 C18 H18B 109.5

H18A C18 H18B 109.5

C16 C18 H18C 109.5

H18A C18 H18C 109.5
H18B C18 H18C 109.5

C20 C19 C21 109.5(4)

C20 C19 Si2 116.0(3)

C21 C19 Si2 118.4(3)

C20 C19 H19 103.6

C21 C19 H19 103.6

Si2 C19 H19 103.6

C19 C20 H20A 109.5

C19 C20 H20B 109.5

H20A C20 H2OB 109.5

C19 C20 H20C 109.5

H20A C20 H20C 109.5

H20B C20 H20C 109.5

C19 C21 H21A 109.5

C19 C21 H21B 109.5

H21A C21 H21B 109.5

C19 C21 H21C 109.5

H21A C21 H21C 109.5

H21B C21 H21C 109.5

C23 C22 C24 109.3(4)

C23 C22 Si2 114.0(3)

C24 C22 Si2 110.2(3)

C23 C22 H22 107.7

C24 C22 H22 107.7

$\mathrm{Si} 2 \mathrm{C} 22 \mathrm{H} 22107.7$

C22 C23 H23A 109.5

C22 C23 H23B 109.5

H23A C23 H23B 109.5

C22 C23 H23C 109.5

H23A C23 H23C 109.5

H23B C23 H23C 109.5

C22 C24 H24A 109.5

C22 C24 H24B 109.5

H24A C24 H24B 109.5

C22 C24 H24C 109.5

H24A C24 H24C 109.5

H24B C24 H24C 109.5

C26 C25 C27 109.7(4)

C26 C25 Si3 112.5(4)

C27 C25 Si3 114.5(3)

C26 C25 H25 106.5

$\mathrm{C} 27 \mathrm{C} 25 \mathrm{H} 25106.5$

Si3 C25 H25 106.5

C25 C26 H26A 109.5

C25 C26 H26B 109.5

H26A C26 H26B 109.5

C25 C26 H26C 109.5

H26A C26 H26C 109.5

H26B C26 H26C 109.5 
C25 C27 H27A 109.5 C25 C27 H27B 109.5 H27A C27 H27B 109.5 C25 C27 H27C 109.5 H27A C27 H27C 109.5 H27B C27 H27C 109.5 C29 C28 C30 109.3(4) C29 C28 Si3 119.1(3) C30 C28 Si3 112.4(3) C29 C28 H28 104.9 C30 C28 H28 104.9 Si3 C28 H28 104.9 C28 C29 H29A 109.5 C28 C29 H29B 109.5 H29A C29 H29B 109.5 C28 C29 H29C 109.5 H29A C29 H29C 109.5 H29B C29 H29C 109.5 C28 C30 H30A 109.5 C28 C30 H30B 109.5 H30A C30 H30B 109.5 C28 C30 H30C 109.5 H30A C30 H30C 109.5 H30B C30 H30C 109.5 C32 C31 C33 110.7(3) C32 C31 Si3 111.6(3) C33 C31 Si3 112.7(3) C32 C31 H31 107.2 C33 C31 H31 107.2 Si3 C31 H31 107.2 C31 C32 H32A 109.5 C31 C32 H32B 109.5 H32A C32 H32B 109.5 C31 C32 H32C 109.5 H32A C32 H32C 109.5 H32B C32 H32C 109.5 C31 C33 H33A 109.5 C31 C33 H33B 109.5 H33A C33 H33B 109.5 C31 C33 H33C 109.5 H33A C33 H33C 109.5 H33B C33 H33C 109.5 Sn1 C34 H34A 109.5 Sn1 C34 H34B 109.5 H34A C34 H34B 109.5 Sn1 C34 H34C 109.5 H34A C34 H34C 109.5 H34B C34 H34C 109.5 Sn1 C35 H35A 109.5
Sn1 C35 H35B 109.5 H35A C35 H35B 109.5 Sn1 C35 H35C 109.5 H35A C35 H35C 109.5 H35B C35 H35C 109.5 Sn1 C36 H36A 109.5 Sn1 C36 H36B 109.5 H36A C36 H36B 109.5 Sn1 C36 H36C 109.5 H36A C36 H36C 109.5 H36B C36 H36C 109.5 C2 N1 Si1 117.2(2) C2 N1 U1 113.0(2) Si1 N1 U1 129.84(14) C4 N2 Si2 115.1(2) C4 N2 U1 114.3(2) Si2 N2 U1 130.57(15) C6 N3 Si3 116.8(2) C6 N3 U1 113.2(2) Si3 N3 U1 129.98(15)

C5 N4 C3 111.7(3) C5 N4 C1 111.0(3) C3 N4 C1 111.1(3) C5 N4 U1 107.41(19)

C3 N4 U1 108.1(2) C1 N4 U1 107.28(19) N1 Si1 C10 106.82(14) N1 Si1 C7 111.50(14) C10 Si1 C7 107.76(15) N1 Si1 C13 111.43(15) C10 Si1 C13 113.91(17) C7 Si1 C13 105.44(15) N2 Si2 C16 106.54(16) N2 Si2 C22 110.65(17) C16 Si2 C22 109.3(2) N2 Si2 C19 115.18(17) C16 Si2 C19 106.8(2) C22 Si2 C19 108.20(19) N3 Si3 C25 105.28(16) N3 Si3 C31 111.99(14) C25 Si3 C31 109.2(2) N3 Si3 C28 111.67(18) C25 Si3 C28 113.7(2) C31 Si3 C28 105.13(18) C35 Sn1 C34 97.25(17) C35 Sn1 C36 97.23(17) C34 Sn1 C36 98.41(18) C35 Sn1 U1 120.17(11) C34 Sn1 U1 117.65(12) 
C36 Sn1 U1 121.12(12)

N3 U1 N2 110.15(10)

N3 U1 N1 108.77(10)

N2 U1 N1 109.47(10)

N3 U1 N4 70.94(10)

N2 U1 N4 70.06(10)
N1 U1 N4 70.57(9)

N3 U1 Sn1 110.40(7)

N2 U1 Sn1 109.33(7)

N1 U1 Sn1 108.70(7)

N4 U1 Sn1 178.66(7) 
Crystallographic Information for $\mathrm{LiUCl}_{4}(\mathrm{pyr})_{7}(3)$.
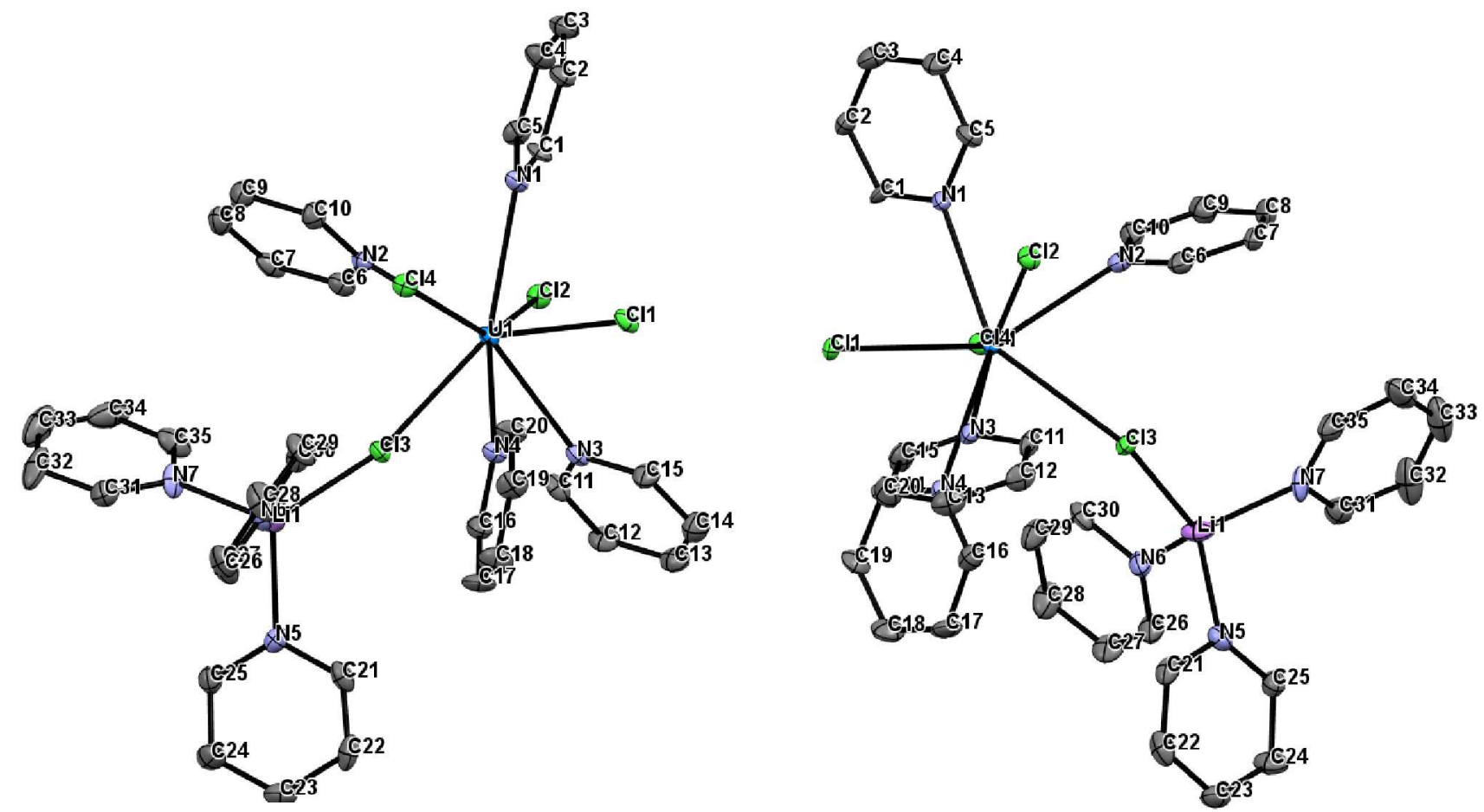

Figure S7. Thermal ellipsoid representations of $\mathbf{3}$ at the $50 \%$ probability level, from two perspectives. 
Table S5. Crystal parameters for 3.

\begin{tabular}{|c|c|c|c|c|}
\hline Bond precision: & $C-C=0.0173 \mathrm{~F}$ & & Wavelength $=$ & 0.71073 \\
\hline \multirow[t]{2}{*}{ Cell: } & $a=17.762(4)$ & & $=11.798(3)$ & $C=19.524(4)$ \\
\hline & alpha $=90$ & & et $a=113.735(2)$ & gamma $=90$ \\
\hline \multirow[t]{2}{*}{ Temperature: } & $100 \mathrm{~K}$ & & & \\
\hline & Calculated & & Reported & \\
\hline Volume & $3745.3(15)$ & & $3745.3(14)$ & \\
\hline Space group & P 21/c & & P2 (1)/c & \\
\hline Hall group & $-\mathrm{P} 2 \mathrm{ybc}$ & & $?$ & \\
\hline Moiety formula & C35 H35 Cl4 Li & N7 & $\mathrm{U}$ & \\
\hline Sum formula & C35 H35 Cl4 Li & N7 & C35 H35 Cl & 4 Li N7 U \\
\hline Mr & 940.47 & & 940.45 & \\
\hline $\mathrm{Dx}, \mathrm{g} \mathrm{cm-3}$ & 1.668 & & 1.668 & \\
\hline $\mathrm{z}$ & 4 & & 4 & \\
\hline Mu $(m m-1)$ & 4.654 & & 4.654 & \\
\hline F000 & 1828.0 & & 1828.0 & \\
\hline F000' & 1792.39 & & & \\
\hline $\mathrm{h}, \mathrm{k}, \mathrm{lmax}$ & $23,15,26$ & & $23,15,26$ & \\
\hline Nref & 9302 & & 9257 & \\
\hline $\operatorname{Tmin}, \operatorname{Tmax}$ & $0.458,0.498$ & & $0.645,0.74$ & \\
\hline Tmin' & 0.449 & & & \\
\hline \multicolumn{2}{|c|}{$\begin{array}{l}\text { Correction method= \# Reported } \mathrm{T} \\
\text { AbsCorr }=\text { EMPIRICAL }\end{array}$} & T Li & imits: $\operatorname{Tmin}=0.645 \mathrm{Tn}$ & $\max =0.746$ \\
\hline \multicolumn{2}{|c|}{ Data completeness $=0.995$} & \multicolumn{3}{|c|}{ Theta $(\max )=28.280$} \\
\hline $\mathrm{R}($ reflections $)=$ & $0.0645(7381)$ & & $\mathrm{wR} 2($ reflections $)=$ & $0.1629(9257)$ \\
\hline$S=1.278$ & Npar: & & 33 & \\
\hline
\end{tabular}

Table S6. Bond lengths for 3.

U1 N1 2.739(8)

U1 N2 2.772(8)

U1 N4 2.776(8)

U1 N3 2.784(9)

U1 Cl2 2.793(2)

U1 Cl4 2.804(2)

U1 Cl1 2.803(2)

U1 $\mathrm{Cl} 32.904(2)$
N5 C21 1.322(14)

N5 C25 1.351(14)

N5 Li1 2.08(2)

C23 C24 1.377(16)

C23 C22 1.403(16)

C25 C24 1.380(15)

C21 C22 1.362(16)

N3 C15 1.329(13) 
N3 C11 1.364(12)

N1 C5 1.355(13)

N1 C1 1.335(12)

N2 C10 1.335(13)

N2 C6 1.355(13)

C8 C7 1.372(17)

C8 C9 1.387(16)

C6 C7 1.390(15)

C9 C10 1.382(15)

C12 C11 1.367(14)

C12 C13 1.383(16)

C15 C14 1.381(16)

C14 C13 1.378(17)

N7 C35 1.317(15)

N7 C31 1.343(14)

N7 Li1 2.04(2)

C31 C32 1.383(16)

C34 C33 1.36(2)

C34 C35 1.378(17)

C33 C32 1.40(2)

C2 C3 1.384(15)

C2 C1 1.397(13)

C5 C4 1.384(15)

C3 C4 1.392(16)

N4 C16 1.346(13)

N4 C20 1.347(12)

C16 C17 1.397(15)

C20 C19 1.400(13)

C18 C19 1.371(16)

C18 C17 1.384(16)

N6 C30 1.369(14)

N6 C26 1.347(14)

N6 Li1 2.01(2)

C27 C26 1.361(16)

C27 C28 1.391(17)

C30 C29 1.371(15)

C29 C28 1.372(17)

Cl3 Li1 2.352(18)

Table S7. Bond angles for 3.

N1 U1 N2 72.9(2)

N1 U1 N4 131.0(2)

N2 U1 N4 130.2(2)

N1 U1 N3 131.0(2)

N2 U1 N3 129.5(2)

N4 U1 N3 72.6(2)

N1 U1 Cl2 76.55(18)

N2 U1 Cl2 76.38(18)
N4 U1 Cl2 142.89(18)

N3 U1 Cl2 70.31(18)

N1 U1 Cl4 74.22(18)

$\mathrm{N} 2 \mathrm{U} 1 \mathrm{Cl} 4$ 76.22(18)

N4 U1 Cl4 72.53(18)

N3 U1 Cl4 145.09(18)

$\mathrm{Cl} 2$ U1 Cl4 144.57(7)

N1 U1 Cl1 73.69(18)

N2 U1 Cl1 146.21(18)

N4 U1 Cl1 71.61(18)

N3 U1 Cl1 77.85(18)

$\mathrm{Cl} 2$ U1 Cl1 100.34(7)

Cl4 U1 Cl1 90.27(7)

N1 U1 Cl3 144.64(18)

$\mathrm{N} 2 \mathrm{U} 1 \mathrm{Cl} 3$ 71.78(17)

N4 U1 Cl3 75.03(17)

N3 U1 Cl3 74.32(17)

$\mathrm{Cl} 2 \mathrm{U} 1 \mathrm{Cl} 3$ 94.75(7)

Cl4 U1 Cl3 97.58(7)

Cl1 U1 Cl3 141.53(7)

C21 N5 C25 118.6(9)

C21 N5 Li1 119.6(9)

C25 N5 Li1 118.1(9)

C24 C23 C22 118.2(10)

N5 C25 C24 121.4(10)

N5 C21 C22 123.5(10)

C23 C24 C25 119.6(11)

C21 C22 C23 118.6(10)

C15 N3 C11 115.5(9)

C15 N3 U1 123.9(7)

C11 N3 U1 119.8(7)

C5 N1 C1 116.8(9)

C5 N1 U1 123.8(7)

C1 N1 U1 119.0(6)

C10 N2 C6 116.9(9)

C10 N2 U1 125.6(7)

C6 N2 U1 116.1(7)

C7 C8 C9 118.1(10)

N2 C6 C7 122.5(10)

C8 C9 C10 119.1(11)

N2 C10 C9 123.6(10)

C8 C7 C6 119.7(10)

C11 C12 C13 120.4(10)

N3 C15 C14 124.8(10)

C13 C14 C15 119.0(11)

N3 C11 C12 123.0(10)

C35 N7 C31 117.6(10)

C35 N7 Li1 121.2(10)

C31 N7 Li1 121.2(9) 
C14 C13 C12 117.3(10)

N7 C31 C32 123.3(12)

C33 C34 C35 118.8(13)

N7 C35 C34 123.8(13)

C34 C33 C32 119.1(12)

C33 C32 C31 117.4(13)

C3 C2 C1 118.5(10)

N1 C5 C4 122.9(10)

C2 C3 C4 118.2(10)

N1 C1 C2 124.0(10)

C5 C4 C3 119.5(10)

C16 N4 C20 117.3(9)

C16 N4 U1 125.0(7)

C20 N4 U1 117.6(6)

N4 C16 C17 123.0(10)

N4 C20 C19 122.7(9)

C19 C18 C17 119.0(10)
C16 C17 C18 118.7(10)

C20 C19 C18 119.3(10)

C30 N6 C26 116.8(10)

C30 N6 Li1 115.8(9)

C26 N6 Li1 127.3(10)

C26 C27 C28 119.0(11)

N6 C30 C29 122.0(10)

C27 C26 N6 123.6(11)

C30 C29 C28 120.0(11)

C27 C28 C29 118.4(11)

Li1 Cl3 U1 139.8(5)

N6 Li1 N7 108.4(9)

N6 Li1 N5 100.0(9)

N7 Li1 N5 108.1(10)

N6 Li1 Cl3 112.5(9)

N7 Li1 Cl3 111.0(8)

N5 Li1 Cl3 116.2(8)

References

1. Zhao, Y.; Truhlar, D. G. Theor. Chem. Acc. 2008, 120, 215. 